\title{
Revisión sistemática de la producción española sobre rendimiento académico entre 1980 y 2011
}

\section{Systematic review of the Spanish production about academic performance between 1980 and 2011}

\author{
Lidia CABRERA PÉREZ \\ Universidad de La Laguna
}

Recibido: Mayo 2014

Evaluado: Julio 2014

Aceptado: Octubre 2015

\section{Resumen}

El bajo rendimiento académico sigue siendo un problema de investigación de gran impacto social. Sin embargo, los resultados científicos no están teniendo repercusión en la mejora, ni en las políticas educativas. En este trabajo nos propusimos identificar, ordenar, analizar y extraer conclusiones sobre la producción española sobre rendimiento académico, con el fin de visualizarla y derivar problemas futuros objeto de investigación. Para ello se llevó a cabo un estudio de revisión en seis etapas, en el que se combinaron procedimientos metodológicos de investigación de síntesis y de investigación bibliométrica. Se identificaron las publicaciones en cuatro bases de datos españolas; posteriormente se analizaron y codificaron los datos extraídos alrededor de nueve indicadores: título, año, tipo de documento, editorial, autor, número autores, institución, comunidad autónoma y tema. Los resultados muestran una producción constante y creciente (1595 referencias), pero dispersa, tanto por el inmenso número de autores que publican, la mayoría con un solo trabajo, como por los lugares donde publican. Las temáticas más tratadas se refieren en primer lugar a modelos explicativos, diagnóstico y tratamiento; en segundo lugar se abordan los factores y variables determinantes del bajo y alto rendimiento académico; en tercer lugar se desarrollan estudios sobre legislación y de otras variables psicológicas y educativas en relación con el rendimiento. Este estudio supone una aproximación sintetizada de la producción sobre rendimiento académico en España, y un primer paso para investigaciones de síntesis posteriores, que nos permitan identificar buenas prácticas para el ejercicio de "prácticas basadas en la evidencia”, y otros problemas de investigación.

Palabras clave: rendimiento académico, fracaso escolar, éxito académico, revisión sistemática, investigación de síntesis, estudio bibliométrico.
Abstract
Low Academic performance is a research problem of great social impact. However, scientific findings are not having an impact in terms of improvements or on educational policies. In this paper, our goals were to identify, organize, analyze and draw conclusions regarding Spanish academic performance in order to visualize the problems and identify areas for future research. This was carried out as a six-step procedure, in which methodological procedures using 
synthesis and bibliometric research were combined. Publications were identified in four Spanish databases; the data was then analyzed and coded under nine indicators: title, year, type of paper, publisher, author, number of authors, institution, region and topic. The results show steadily increasing production (1595 references), but disperse in terms of the huge number of published authors, most with a single paper, and of the places where they were published. The topics most widely addressed were explanatory models, diagnosis and treatment, followed by the factors and variables that determined the high or low academic achievement and, thirdly, studies of legislation and other performance-related psychological and educational variables. This study is a synthesis of production about academic performance in Spain and is a first step towards further synthesis research, to identify best practices for the exercise of "evidence-based practices”, and other research problems.

Keywords: Academic performance, school failure, academic success, sistematic review, research synthesis, bibliometric study.

El rendimiento académico y las dificultades de logro del alumnado siguen siendo un problema de investigación de gran impacto social, y los informes de evaluación y diagnóstico educativo que se hacen a escala nacional e internacional se centran básicamente en esos resultados. Sin embargo, las conclusiones de investigación no están teniendo repercusiones en la mejora, ni en las políticas educativas. Con frecuencia, las acciones educativas obvian los modelos y hallazgos de estudios. ¿Cuál es la razón?, ¿no está ofreciendo la investigación y la teoría educativa respuestas a las necesidades reales?, o ¿está el conocimiento educativo tan oculto y disperso que no alcanza su aplicación? Con este trabajo nos propusimos identificar, ordenar, analizar y extraer conclusiones de la producción española sobre rendimiento académico, con el fin de visualizarla y derivar problemas futuros objeto de investigación.

El crecimiento exponencial de la literatura científica, así como las necesidades de credibilidad, transparencia y compromiso social, han requerido que la ciencia sea medida, sintetizada y evaluada. Estos resultados facilitan la toma de decisiones económicas, políticas y profesionales (Fernández Cano, 1995; Maltrás, Quintanilla y Vidal, 1998; Sánchez Meca y Botella, 2010). Harris Cooper (2009) muestra en su libro sobre "Research synthesis and meta-analysis", como las grandes decisiones de prácticos, teóricos y asesores políticos para la mejora y el bienestar humano han estado basadas en "investigaciones de síntesis”.

El primer peldaño en la medición y evaluación de la investigación lo ha de constituir la bibliometría, ya que es ésta la que nos permite determinar el estado del conocimiento de un área o tópico objeto de investigación, requisito imprescindible para determinar finalidades posteriores de evaluación y síntesis (cienciometria, metaanálisis, síntesis, etc.). Por eso, este trabajo constituye sólo el primer peldaño necesario en la investigación de síntesis sobre rendimiento académico.

La bibliometría, en sus inicios, se limitaba a medir los datos bibliográficos, pero en las últimas décadas ha alcanzado un gran desarrollo, gracias al uso de técnicas estadísticas sofisticadas y bases de datos informatizadas que han servido de soporte al estudio del tamaño, crecimiento y distribución de las publicaciones, en la valoración de la calidad investigadora, y en el estudio de la estructura y desarrollo de las disciplinas 
científicas y los grupos de investigadores que la producen y consumen. La bibliometría se ha implantado como técnica metodológica imprescindible para la evaluación de la producción científica y todos los fenómenos ligados a la comunicación de la ciencia, atribuyéndosele finalidades tales como las de: 1) revisar los métodos de las investigaciones publicadas; 2) verificar la situación de diversas áreas de conocimiento; 3) revisar el valor de las publicaciones; 4) evaluar la relación entre los autores y sus publicaciones; 5) calificar y determinar el impacto de las publicaciones científicas y el valor que representan (Quevedo-Blasco y López-López, 2010).

Las bondades atribuidas a la bibliometría son: 1) evaluar la productividad de autores e instituciones o grupos colaboradores y su evolución temporal; 2) realizar mapas de colaboración en cuanto a la distribución de autores por número de trabajos publicados y la detección de grupos o “colegios invisibles”; 3) Conocer el índice de especialización científica de un país: qué áreas de conocimiento son más destacadas en el ámbito nacional y cuál es el peso relativo en el panorama internacional; 4) representar la estructura básica de la bibliografía de una disciplina mediante el análisis de referencias y citas; 5) conocer las publicaciones con más prestigio e impacto (López-López, 1996; López-López y Tortosa Gil, 2002).

Los indicadores bibliométricos, sin embargo, son los que determinan el fin último de cada estudio de medición y evaluación de la ciencia, determinando qué información cuantitativa y cualitativa será objeto de análisis y valoración. Normalmente se distingue entre los indicadores que miden la productividad y los que evalúan la calidad científica de los trabajos. En este sentido, los indicadores bibliométricos no sólo permiten apoyar decisiones en datos objetivos como las "Informed peer review" (Koening, 1983; Nederhof y Van Raan, 1987; Warner 2000), sino detectar perfiles temáticos de actividad, identificar fortalezas, analizar colaboraciones, estudiar tendencias, o identificar sesgos en el avance de la ciencia.

Pero los estudios bibliométricos siempre han estado más orientados a la producción y al impacto (Camps, 2008; López Piñero y Terrada, 1993) que al estudio del proceso de construcción de la ciencia y la calidad de sus resultados. Esta ha sido más una finalidad de los estudios de síntesis y metaanalíticos (Cooper, 2009).

Las necesidades de la práctica educativa requieren trabajos de revisión que identifiquen y sinteticen hallazgos útiles para la mejora de los resultados educativos, así como trabajos de evaluación de la calidad que identifiquen la adecuación y pertinencia de los problemas de investigación, rigor metodológico con el que son tratados, áreas de relevancia a las que no se les ha prestado atención, etc. para lograr un mayor impacto. En ese marco, identificar la producción científica constituye la tarea preliminar, por lo que decidimos realizar este estudio del conocimiento científico sobre rendimiento académico a través de una revisión de corte bibliométrico, con la finalidad de identificar y caracterizar la producción total, indispensable para estudios posteriores de las características reseñadas. Derivados de esta finalidad, los indicadores de análisis utilizados fueron más propios de la investigación bibliométrica que de la investigación de síntesis y metaanalítica, basados en el recuento de las publicaciones y elementos bibliográficos, y dentro de estos, atendiendo más a indicadores de producción que de colaboración y circulación. En esta decisión se tuvieron en cuenta las sugerencias de 
Peña Ocando, Portillo y Caldera (2011), que identifican dos niveles en la investigación bibliométrica: un primer nivel básico-diagnóstico, y otro relacional inferencial, más profundo y a partir del cual se puede producir y deducir hallazgos más relevantes, que "informan acerca de las relaciones entre los productores y agentes científicos en el proceso que ha concluido con la publicación conjunta de resultados” (Peña Ocando, Portillo y Caldera, 2011, pg.5).

\section{Método}

El diseño de la investigación constituye una combinación metodológica entre procedimientos propios de la investigación de síntesis y meta-analítica y los específicos de los estudios bibliométricos.

Siguiendo los modelos tradicionales de diseño de investigación de síntesis (Taveggia, 1974; Cooper, 2007) y bibliométricos (López-López, 1996; Persson, Glänzel y Danell, 2004) se llevaron a cabo seis etapas: 1) formulación del problema; 2) investigación de la literatura, para identificar y seleccionar las fuentes de información; 3) recolección de la información de los estudios; 4) Análisis e integración de los resultados; 5) Interpretación de las evidencias; 6) Presentación de los resultados. La "evaluación de la calidad de los estudios", etapa introducida por Cooper en obras posteriores (2009), no se tuvo en cuenta en este estudio.

\section{Etapa 1. Planteamiento del problema y objetivos}

Los objetivos de esta investigación fueron identificar y analizar la producción científica española sobre rendimiento académico (publicaciones científicas) entre los años 1980-2011, y caracterizarla atendiendo a indicadores bibliométricos de producción científica y de síntesis de la investigación. Para ello se propusieron los siguientes objetivos específicos:

- Identificar y localizar la producción científica sobre rendimiento académico en España a partir de los documentos científicos publicados en el intervalo de tiempo 1980-2011.

- Analizar y clasificar dicha producción de acuerdo a indicadores bibliométrico de producción científica.

- Conocer tendencias de producción y problemas objeto de estudio en el periodo analizado.

- Identificar grupos de investigación pioneros, y su procedencia.

\section{Etapa 2: Búsqueda e identificación de las fuentes documentales}

La búsqueda documental se realizó utilizando descriptores seleccionados entre los propuestos por tesauros internacionales especializados en educación, y se definieron los criterios de inclusión y exclusión de documentos en la muestra. 
- Criterios de identificación. Se analizaron el Tesauro de la Educación Europea (TEE) y el Tesauro de la UNESCO. Se seleccionaron descriptores que incluyeran los dos polos del progreso académico (éxito y fracaso). Resultaron seleccionados los siguientes: "Rendimiento académico", "Rendimiento escolar", "Fracaso escolar", "Fracaso académico" y "Éxito escolar". Las bases de datos consultadas fueron las siguientes: DIALNET, ISOC, TESEO e ISBN.

- Criterios de inclusión. Se determinó incluir: a) todos los documentos científicos teóricos y empíricos publicados en editoriales y revistas españolas, referidos tanto a estudios nacionales como de otra procedencia; b) todos los documentos publicados en el período de tiempo comprendido entre los años 1980-2011; c) que el contenido de los documentos versara sobre rendimiento académico, y en los que éste fuera el eje central del estudio.

\section{Selección e identificación de la muestra}

El procedimiento consistió en identificar y seleccionar los documentos, tras excluir los que se iban repitiendo y los que no cumplían los criterios de inclusión. En la tabla I se muestra el número de documentos identificados en cada base de datos, así como el número de excluidos e incluidos.

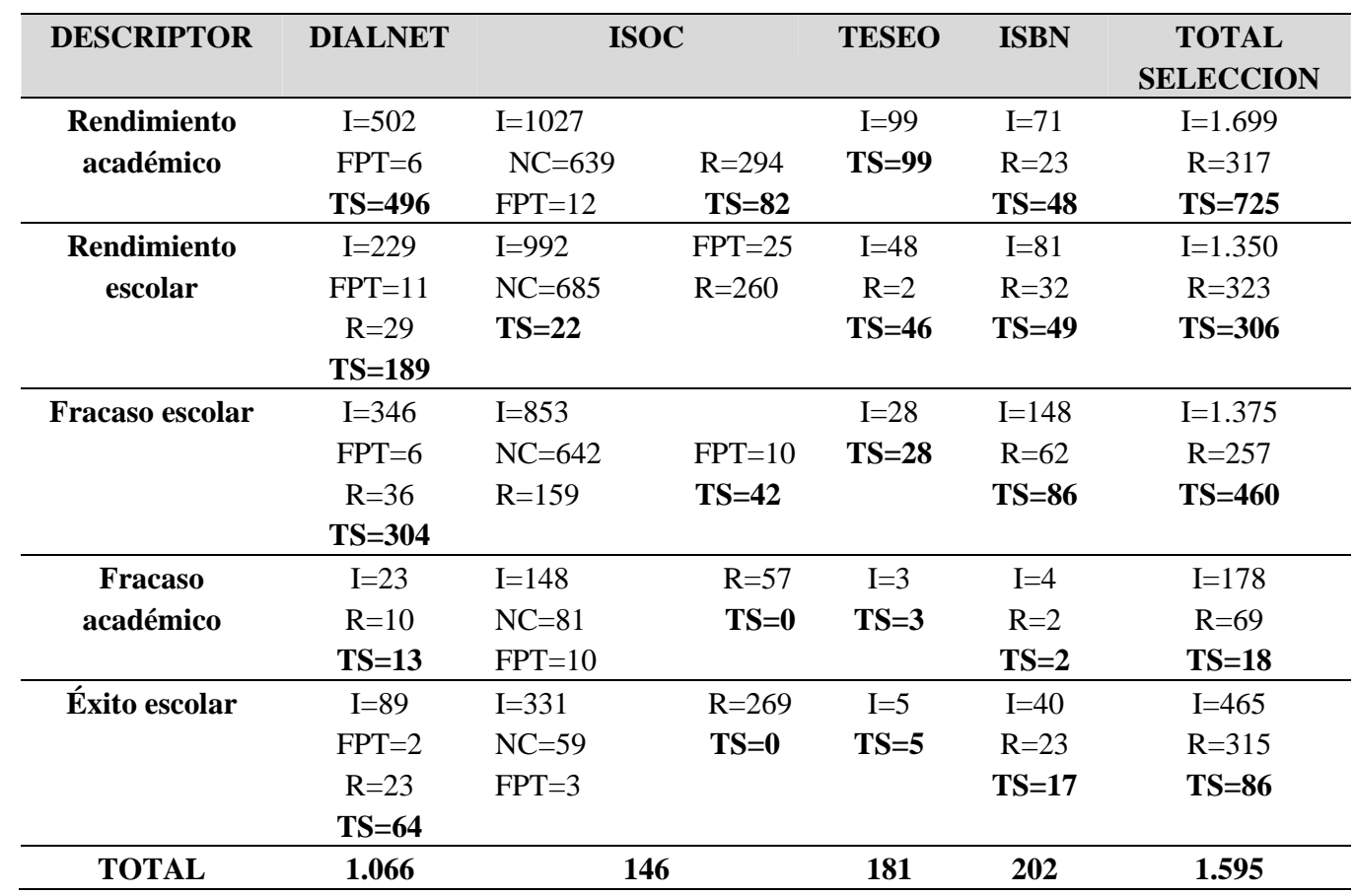

I=Documentos identificados tras la búsqueda con cada descriptor; FPT=Fuera del periodo temporal; $\mathrm{R}=$ Ya identificados y seleccionados en búsquedas anteriores; OT=Otra temática y no resultados de investigación; TS=Total seleccionados.

Tabla I. $\mathrm{N}^{\circ}$ de documentos identificados, incluidos y excluidos, por descriptor y base de datos. 
La muestra quedó constituida por 1.595 unidades de análisis, entre tesis y documentos de investigación publicados en libros y revistas nacionales.

\section{Etapa 3: Recolección de información de los estudios: Codificación y análisis}

Para esta tarea tuvimos que definir previamente la información que necesitábamos recoger de cada una de las unidades de análisis, y posteriormente construir una herramienta que nos permitiera codificar y registrar los datos extraídos.

\section{Selección y descripción de indicadores objeto de análisis}

Atendiendo a nuestras finalidades, el conjunto de indicadores de análisis fueron:

- Título del Documento: como indicador de foco temático y de lo nuevo y relevante que aporta el trabajo.

- Año de publicación: con la finalidad de analizar la evolución de la producción en el tiempo.

- Tipo de documento: indicador internacional referido al soporte de difusión, a la vez que indicativo de la visibilidad y competitividad de la producción, en función del ranking que ocupa cada revista y editorial.

- Editorial: con el fin de analizar el rigor y calidad de la producción, en función de revisiones externas de la fuente, así como otros criterios de rigor de la revista o editorial.

- Nombre del Autor/es: nos permite identificar autores pioneros, definir la cantidad de autores que han producido resultados en un período determinado, etc.

- Número de autores: con el fin de averiguar si los investigadores constituyen unidades aisladas o equipos de trabajo y explorar sobre la evolución de la producción de un sistema en relación con el número medio de firmantes de los trabajos.

- Institución: para averiguar desde dónde y por quién se hace la investigación.

- Comunidad autónoma: nos permite localizar geográficamente a los investigadores, las zonas de mayor producción, y posteriormente indicadores relacionados con dicha producción, como el número de universidades o la magnitud de las mismas; financiación de cada comunidad a la investigación; importancia política otorgada al tema; etc.

- Tema de investigación: para determinar las temáticas más relevantes o tenidas en cuenta por los investigadores en sus estudios sobre rendimiento académico.

En la siguiente tabla se muestra la relación de indicadores y subindicadores que constituyeron la base para el análisis de los documentos y codificación de datos extraídos. 


\begin{tabular}{ll}
\hline \multicolumn{1}{c}{ INDICADORES } & \multicolumn{1}{c}{ SUBINDICADORES } \\
\hline Título & Título completo (análisis de Descriptores de los Tesauros). \\
Año de publicación & De 1980 a 2011. \\
Tipo de documento & Libro; Capítulo de libro; Artículo de revista; Tesis; Acta de \\
congreso; Otros.
\end{tabular}

Tabla II. Indicadores y subindicadores objeto de análisis.

\section{Diseño de base de datos para la codificación y registro de los datos}

Se diseñó una base de datos relacional utilizando el programa informático Microsoft Access 2007. En una tabla principal se recogió la información contenida en las referencias bibliográficas de cada documento analizado (que constituyó cada registro), desglosada en distintos campos o columnas, que se correspondían con los indicadores de análisis de la investigación.

\section{Análisis de los documentos y registro de información identificada en la base de datos}

En este paso se revisó cada documento, se extrajo información referida a cada uno de los indicadores, y se registró en la base de datos. Posteriormente se procedió al análisis de dicha información a través de la función "consultas”.

\section{Resultados $^{1}$}

Este apartado incluye las tres etapas restantes del procedimiento de investigación: análisis e integración de resultados; interpretación de las evidencias; presentación de

\footnotetext{
${ }^{1}$ El análisis y volcado de datos en la base de datos fue realizado por la becaria Alicia Lázaro González, beneficiaria de una beca para posgraduados de la Universidad de La Laguna para esta investigación, con fondos de la Fundación Caja Canarias.
} 
resultados. Las tres tareas se muestran fusionadas en el tratamiento de los resultados generados en cada descriptor objeto de análisis.

\section{Título del documento}

Lo primero que nos interesó fue comprobar si los descriptores utilizados para la búsqueda figuraban en los títulos de los documentos, y con ello respondían al requerimiento de uso de lenguaje normalizado, que aconsejan tanto La Asociación Española de Normalización y Certificación (AENOR), como las Normas ISO internacionales. Se procedió a contabilizar la frecuencia de aparición en los títulos de cada uno de los descriptores utilizados. Como podemos observar en la tabla III, hay una alta correlación entre el descriptor usado para la identificación del documento y su inclusión en el título, pues sólo 46 documentos no incluyen en sus títulos alguno de los descriptores de búsqueda. El término más frecuentemente usado es el de "rendimiento académico”, y el menos usado el de “fracaso académico”. Sin embargo, con este último descriptor (fracaso académico) se localizaron muchos más artículos que con los otros descriptores. A la inversa sucede con el descriptor "fracaso escolar", muy frecuentemente usado en los títulos, y menos potente para identificar documentos relacionados. En general, los resultados nos permiten atribuir al título de los documentos científicos sobre rendimiento académico un correcto uso de lenguaje normalizado.

\begin{tabular}{|c|c|c|c|c|c|}
\hline Descriptor & $\begin{array}{l}\text { Documentos } \\
\text { identificados }\end{array}$ & $\begin{array}{l}\text { Documentos } \\
\text { repetidos }\end{array}$ & $\begin{array}{l}\text { Documentos } \\
\text { excluidos }\end{array}$ & $\begin{array}{l}\text { Documentos } \\
\text { seleccionados }\end{array}$ & $\begin{array}{l}\text { Incluido } \\
\text { título } \\
\end{array}$ \\
\hline $\begin{array}{l}\text { Rendimiento } \\
\text { académico }\end{array}$ & 1.699 & 317 & 657 & 725 & 667 \\
\hline Rendimiento escolar & 1.350 & 323 & 721 & 306 & 302 \\
\hline Fracaso académico & 1.375 & 257 & 658 & 460 & 26 \\
\hline Fracaso escolar & 178 & 69 & 91 & 18 & 470 \\
\hline Éxito escolar & 465 & 315 & 64 & 86 & 84 \\
\hline \multirow{2}{*}{\multicolumn{4}{|c|}{ No incluyen estos descriptores en el título }} & +46 & 1.549 \\
\hline & & & & MUESTRA & 1.595 \\
\hline
\end{tabular}

Tabla III. Documentos identificados por cada descriptor y su frecuencia de aparición en el título.

Para identificar otros ámbitos estudiados en relación con el rendimiento, se hizo un recuento de términos más usados en los títulos, resultando un amplio conjunto de variables que analizamos y agrupamos en: a) variables referidas al contexto, a la intervención y a la evaluación; y b) variables referidas a otros constructos relacionados con el rendimiento (Tablas IV y V). Algunos estudios se refieren a más de un tópico, por lo que han sido incluidos en más de una categoría. 


\begin{tabular}{|c|c|c|c|}
\hline Ámbito científico de referencia & Término incluido en el título & $\begin{array}{l}N^{0} \text { Doc } \\
M=1595\end{array}$ & $\%$ \\
\hline \multirow{5}{*}{ CONTEXTO } & Escolar & 904 & 56,6 \\
\hline & Escuela & 33 & 2 \\
\hline & Educación primaria & 26 & 1,6 \\
\hline & Bachillerato & 31 & 1,9 \\
\hline & Educación Superior & 8 & 0,5 \\
\hline \multirow{6}{*}{$\begin{array}{l}\text { INTERVENCIÓN Y ENSEÑANZA } \\
\text { APRENDIZAJE }\end{array}$} & TOTAL & 1.002 & 62,8 \\
\hline & Educación & 148 & 9,2 \\
\hline & Aprendizaje & 112 & 7 \\
\hline & Programa & 26 & 1,6 \\
\hline & Tratamiento & 9 & 0,5 \\
\hline & Estrategias & 58 & 3,6 \\
\hline \multirow{10}{*}{$\begin{array}{l}\text { EVALUACIÓN Y FACTORES } \\
\text { CAUSALES }\end{array}$} & Enseñanza & 56 & 3,5 \\
\hline & Formación & 27 & 1,6 \\
\hline & TOTAL & 436 & 27,3 \\
\hline & Evaluación & 56 & 3,5 \\
\hline & Diagnóstico & 7 & 0,4 \\
\hline & Causas & 34 & 2,1 \\
\hline & Atribuciones causales & 23 & 1,4 \\
\hline & Factores de riesgo & 3 & 0,1 \\
\hline & Predicción+prevenir & $29+7$ & 2,2 \\
\hline & TOTAL & 149 & 9,3 \\
\hline
\end{tabular}

Tabla IV. Términos con mayor frecuencia de aparición en los títulos.

\begin{tabular}{llcc}
\hline $\begin{array}{l}\text { Variables relacionadas con el } \\
\text { rendimiento }\end{array}$ & Término incluido en el título & $\begin{array}{c}\mathbf{N}^{\mathbf{0}} \mathbf{D o c} \\
\mathbf{M = 1 5 9 5}\end{array}$ & $\mathbf{\%}$ \\
\hline \multirow{2}{*}{ VARIABLES PERSONALES } & Motivación & 56 & 3,5 \\
& Autoconcepto & 50 & 3,1 \\
& Inteligencia & 46 & 2,8 \\
& Personalidad & 22 & 1,3 \\
& Ansiedad & 18 & 1,1 \\
& Autoestima & 16 & 1 \\
& Estilos de aprendizaje & 16 & 1 \\
& Estilos cognitivos & 3 & 0,1 \\
& Depresión & 6 & 0,3 \\
& Impulsividad & 6 & 0,3 \\
& Hiperactividad & 8 & 0,5 \\
& Inteligencia emocional & 9 & 0,5 \\
& Razonamiento & 3 & 0,1 \\
& Esfuerzo & 4 & 0,2 \\
& TOTAL & $\mathbf{2 6 3}$ & $\mathbf{1 6 , 4}$ \\
\hline
\end{tabular}

Tabla V. Estudios sobre constructos y variables relacionadas con el rendimiento académico. 


\begin{tabular}{llcc}
\hline $\begin{array}{l}\text { Variables relacionadas con el } \\
\text { rendimiento }\end{array}$ & Término incluido en el título & $\begin{array}{c}\mathbf{N}^{\mathbf{0}} \mathbf{\text { Doc }} \\
\mathbf{M = 1 5 9 5}\end{array}$ & $\mathbf{\%}$ \\
\hline \multirow{3}{*}{ VARIABLES SOCIALES Y } & Familia & 55 & 3,4 \\
FAMILIARES & Factores psicosociales & 4 & 0,2 \\
& Alimentación & 3 & 0,1 \\
& TOTAL & $\mathbf{6 2}$ & $\mathbf{3 , 8}$ \\
VARIABLES ESCOLARES & Exclusión & 20 & 1,25 \\
& Integración & 7 & 0,4 \\
& Bilingüismo & 5 & 0,3 \\
& Violencia escolar & 3 & 0,1 \\
& TOTAL & $\mathbf{3 6 0}$ & $\mathbf{2 2 , 5}$ \\
\hline
\end{tabular}

Tabla V. Estudios sobre constructos y variables relacionadas con el rendimiento académico (cont.).

\section{Año de publicación}

Como era de esperar, los años más productivos han sido los últimos años, sobre todo el 2009 y 2010. A pesar de la existencia de cortos periodos de tiempo en los que la producción se estabiliza, e incluso decrece en algún año, se observa que la producción de cada década casi duplica la de su década anterior (ver tabla VI) resultando en la década del 2.000 una producción superior a las de los 90 y 80 juntas. De este modo encontramos una media de trabajos por año en la década de los 80 de 27,6; una media en los 90 de 43,8; y una media en el 2000 de 73,4.

La producción científica en todos los campo del conocimiento ha aumentado exponencialmente en las últimas décadas, sin embargo, el interés creciente por los resultados educativos y las altas cifras de resultados inadecuados, creemos obedece a factores sociales, como: la preocupación y exigencia social por la calidad de la educación; los objetivos nacionales de aumentar las tasas de formación de la población; la necesidad y exigencia de transparencia de los servicios públicos y rendimiento de cuentas; los informes comparativos de resultados educativos tanto nacionales como internacionales; etc. A pesar de la creación de las unidades de evaluación y calidad de la educación de las distintas Comunidades Autónomas, las líneas prioritarias de investigación han sido la identificación de elementos personales y materiales que influyen en los resultados educativos, así como procedimientos y acciones educativas adaptadas a cada época y cada circunstancia para la mejora de la educación. Si tenemos en cuenta que la finalidad última de la producción científica en educación es la mejora de la calidad y los resultados educativos, la producción centrada exclusivamente en rendimiento académico es relativamente baja, comparada con la producción educativa en general. 


\begin{tabular}{cccccc}
\hline \multicolumn{2}{c}{$\mathbf{1 9 8 0}$} & \multicolumn{2}{c}{$\mathbf{1 9 9 0}$} & \multicolumn{2}{c}{$\mathbf{2 0 0 0}$} \\
\hline Año & $\mathbf{N}^{\mathbf{0}}$ Trabajos & Año & $\mathbf{N}^{\mathbf{0}}$ Trabajos & Año & $\mathbf{N}^{\mathbf{0}}$ Trabajos \\
1980 & $8(0,5 \%)$ & 1990 & $61(3,8 \%)$ & 2000 & $54(3,4 \%)$ \\
1981 & $13(0,8 \%)$ & 1991 & $34(2,1 \%)$ & 2001 & $41(2,5 \%)$ \\
1982 & $12(0,7 \%)$ & 1992 & $45(2,8 \%)$ & 2002 & $56(3,5 \%)$ \\
1983 & $23(1,4 \%)$ & 1993 & $46(2,9 \%)$ & 2003 & $66(4,1 \%)$ \\
1984 & $21(1,3 \%)$ & 1994 & $53(3,3 \%)$ & 2004 & $37(2,3 \%)$ \\
1985 & $38(2,3 \%)$ & 1995 & $30(1,9 \%)$ & 2005 & $73(4,5 \%)$ \\
1986 & $43(2,6 \%)$ & 1996 & $34(2,1 \%)$ & 2006 & $63(3,9 \%)$ \\
1987 & $34(2,1 \%)$ & 1997 & $44(2,7 \%)$ & 2007 & $63(3,9 \%)$ \\
1988 & $39(2,4 \%)$ & 1998 & $50(3,1 \%)$ & 2008 & $85(5,3 \%)$ \\
1989 & $45(2,8 \%)$ & 1999 & $41(2,5 \%)$ & 2009 & $129(8 \%)$ \\
\multicolumn{2}{r}{} & & & 2010 & $127(7,9 \%)$ \\
Total=276 trabajos (17’4\%) & Total=438 trabajos (27,4\%) & Total=881 trabajos (55,2\%) \\
\hline
\end{tabular}

Tabla VI. Número de trabajos publicados anualmente.

\section{Tipo de Documento}

Los resultados desvelan que más de la mitad de los documentos, concretamente el 56 ' $4 \%$ (900 documentos), se han publicado en revistas especializadas. El resto, un 43,6\% (695 documentos), constituyen, por orden de frecuencia, libros, tesis doctorales, capítulos de libros y actas de congresos (tabla VII). El análisis de estos resultados constata la gran difusión de conocimiento científico en educación en libros y capítulos de libros.

Sorprende positivamente la gran producción de tesis doctorales con del fracaso académico como parte del problema de investigación (187 tesis). Sin embargo, no hay una buena relación entre la producción de tesis y publicaciones derivadas de las mismas.

\begin{tabular}{|c|c|c|}
\hline Tipo de documentos & $\mathbf{F}$ & $\%$ \\
\hline Artículo de revista & 900 & 56,4 \\
\hline Libro & 300 & 18,9 \\
\hline Tesis doctoral & 187 & 11,9 \\
\hline Capítulo de libro & 106 & 6,5 \\
\hline Acta de Congreso & 98 & 6,1 \\
\hline Otros & 4 & 0,2 \\
\hline TOTAL & 1595 & 100 \\
\hline
\end{tabular}

Tabla VII. Productividad atendiendo al tipo de documento. 


\section{Editorial}

Con la intención de identificar el tipo de revistas y editoriales, así como su nivel de especialización sobre dificultades académicas y resultados educativos, analizamos las editoriales y revistas que han publicado los trabajos que forman parte de este estudio. Los resultados nos muestran una enorme diversidad y dispersión, con 159 editoriales (que publican 300 libros) y 290 revistas (que publican 900 artículos); algunas revistas de otros ámbitos del conocimientos como de economía y lenguas. El análisis de la relación entre el número de documentos y el números de fuentes de publicación muestra una proporción media de 3 artículos por revista y 2,5 libros por editorial. Ante estos datos, se evidencia la ausencia de un espacio divulgativo centralizado de conocimiento específico sobre rendimiento y resultados educativos.

La editorial que más ha publicado resultados de investigación sobre rendimiento académico lo ha hecho con 12 trabajos. En el otro extremo observamos que 102 editoriales de las 159 (casi dos tercios), sólo han publicado un trabajo. En la tabla VIII se muestra la relación de editoriales que más han publicado sobre rendimiento académico, así como la proporción de trabajos publicados por editorial.

\begin{tabular}{|c|c|c|}
\hline Nombre Editorial & $\begin{array}{c}\mathrm{N}^{\circ} \text { libros publicados } \\
\text { por editorial }\end{array}$ & \\
\hline $\begin{array}{l}\text { Ediciones Didácticas y } \\
\text { Pedagógicas }\end{array}$ & 12 libros & \multirow{12}{*}{$\begin{array}{l}12 \text { editoriales han publicado un total } \\
\text { de } 88 \text { libros }\end{array}$} \\
\hline $\begin{array}{l}\text { Servicio Publicaciones U. } \\
\text { Complutense. }\end{array}$ & 11 libros & \\
\hline Editorial Edita & 10 libros & \\
\hline Tutorial Formación, S.L.L & 9 libros & \\
\hline Ministerio de Educación & 8 libros & \\
\hline $\begin{array}{l}\text { U. Nacional de Educación a } \\
\text { Distancia }\end{array}$ & 6 libros & \\
\hline Narcea S.A. Ediciones & 6 libros & \\
\hline Editorial Madrid, S.L & 6 libros & \\
\hline $\begin{array}{l}\text { Ed Elche: Ajuntament d’Elx, } \\
\text { Regidoria d'Educació i Festes }\end{array}$ & 5 libros & \\
\hline $\begin{array}{l}\text { Asociación Difusión del } \\
\text { Conocimiento Educativo }\end{array}$ & 5 libros & \\
\hline $\begin{array}{l}\text { Servicio de publicaciones U. } \\
\text { de Oviedo }\end{array}$ & 5 libros & \\
\hline $\begin{array}{l}\text { Servicio Publicaciones U. } \\
\text { Santiago Comp. }\end{array}$ & 5 libros & \\
\hline 6 editoriales & 4 cada una & 24 libros \\
\hline 8 editoriales & 3 cada una & 24 libros \\
\hline 31 editoriales & 2 cada una & 62 libros \\
\hline 102 editoriales & 1 cada una & 102 libros \\
\hline TOTAL= 159 editoriales & & 300 libros \\
\hline
\end{tabular}

Tabla VIII. Relación de editoriales que más han publicado sobre rendimiento académico. 
Entre las revistas, destacan 9 que han publicado entre 20 y 44 artículos respectivamente, y 12 revistas que publican entre 10 y 20 artículos. El resto incluyen menos de 10 artículos publicados (ver Tabla IX).

\begin{tabular}{lcc}
\hline \multicolumn{1}{c}{ Revista } & $\begin{array}{c}\mathbf{N}^{\mathbf{0}} \\
\text { artículos }\end{array}$ & $\mathbf{\%}$ \\
\hline Revista de Investigación educativa (RIE) & 44 & 4,9 \\
Revista Galego-Portuguesa de Psicoloxia e Educación & 36 & 4,0 \\
Revista de Psicología general y aplicada & 30 & 3,3 \\
Revista Iberoamericana de Educación & 27 & 3,0 \\
Bordón. Revista de Pedagogía & 25 & 2,7 \\
Revista Electrónica interuniversitaria de formación del profesorado & 25 & 2,7 \\
Cuadernos de Pedagogía & 24 & 2,6 \\
Revista de Ciencias de la Educación & 22 & 2,5 \\
Enseñanza \& Teaching: Revista interuniversitaria de didáctica & 20 & 2,2 \\
Revista Española de Pedagogía & 17 & 1,9 \\
Organización y Gestión Educativa & 15 & 1,6 \\
Revista de Educación & 13 & 1,4 \\
Psicothema & 13 & 1,4 \\
Revista de psicología de la educación & 13 & 1,4 \\
Educadores & 12 & 1,3 \\
Electronic journal of research in educational psychology & 12 & 1,3 \\
& 348 & $53,3 \%$ \\
\hline
\end{tabular}

Tabla IX. Relación de las 16 Revistas que más han publicado.

El conjunto de los datos nos muestra que 31 revistas (10’6\%) publican el 53,3\% de los artículos, mientras que 169 revistas (58’2\%) han publicado un sólo trabajo, el 18'7\% de los artículos; 54 revistas publican sólo 2 artículos y, el resto, 36 revistas (12’4\%), publican entre 3 y 7 trabajos.

\section{Autor}

Este análisis nos permitió identificar autores pioneros, número total de investigadores implicados en estos estudios, y otras características como la continuidad en el tema de investigación y la agrupación entre autores.

El total de autores implicados en la producción de trabajos analizados es de 1.941 (Tabla X). El 82,2\% de estos autores (1.595 autores) sólo han publicado 1 trabajo, y el 11\% (215 autores) han publicado dos trabajos. Por tanto, sólo un 8\% (131 autores, alta y media producción) se caracteriza por ser autores más o menos constantes en la investigación sobre rendimiento académico, entre los cuales destacan 10 autores con más de 9 trabajos (ver Tabla X). 


\begin{tabular}{|c|c|c|c|c|c|}
\hline $\begin{array}{c}\text { Autores } \\
\text { producción alta } \\
\text { (más de } 9 \text { trabajos) } \\
10 \text { autores }(0,5 \%)\end{array}$ & $\begin{array}{l}\text { Número } \\
\text { trabajos/ } \\
\text { autor }\end{array}$ & $\begin{array}{c}\text { Autores } \\
\text { Producción } \\
\text { media (entre } 8 \text { y } \\
3 \text { trabaj) } \\
121 \text { autores } \\
(6,2 \%)\end{array}$ & $\begin{array}{l}\text { Número } \\
\text { trabajos/ } \\
\text { autor }\end{array}$ & $\begin{array}{c}\text { Autores } \\
\text { producción } \\
\text { baja (1 y } 2 \\
\text { trabajos) } \\
1.810 \text { autores } \\
(93,2 \%) \\
\end{array}$ & $\begin{array}{c}\mathrm{N}^{\circ} \\
\text { trabajos/ } \\
\text { autor }\end{array}$ \\
\hline J.L. Castejón Costa & 22 trabajos & 5 autores $(0,2 \%)$ & $\begin{array}{l}8 \text { trabajos } \\
\text { /autor }\end{array}$ & $\begin{array}{l}215 \text { autores } \\
(11 \%)\end{array}$ & $\begin{array}{c}2 \text { trabajos } \\
\text { /autor }\end{array}$ \\
\hline A. Barca Lozano & 16 trabajos & 5 autores $(0,2 \%)$ & $\begin{array}{l}7 \text { trabajos } \\
\text { /autor }\end{array}$ & $\begin{array}{l}1.595 \text { autores } \\
(82,1 \%)\end{array}$ & 1 trabajo \\
\hline $\begin{array}{l}\text { Á. Vázquez Alonso } \\
\text { Antonio Valle Arias }\end{array}$ & 15 trabajos & 12 autores $(0,6 \%)$ & $\begin{array}{l}6 \text { trabajos } \\
\text { /autor }\end{array}$ & & \\
\hline $\begin{array}{l}\text { J. C. Brenlla Blanco } \\
\text { M. Peralbo } \\
\text { Uzquiano }\end{array}$ & 13 trabajos & 14 autores $(0,7 \%)$ & $\begin{array}{l}5 \text { trabajos } \\
\text { /autor }\end{array}$ & & \\
\hline $\begin{array}{l}\text { R. González } \\
\text { Cabanach } \\
\text { J. C. Núñez Pérez }\end{array}$ & 11 trabajos & 28 autores $(1,4 \%)$ & $\begin{array}{l}4 \text { trabajos } \\
\text { /autor }\end{array}$ & & \\
\hline A. M. Porto Riboo & 10 trabajos & 57 autores $(2,9 \%)$ & $\begin{array}{l}3 \text { trabajos } \\
\text { /autor }\end{array}$ & & \\
\hline L. Navas Martínez & 9 trabajos & & & & \\
\hline \multicolumn{4}{|c|}{ 1.941 AUTORES=1.595 TRABAJOS } & & \\
\hline
\end{tabular}

Tabla X. Número trabajos publicados por autor.

\section{Número de autores por trabajo}

Este indicador tiene la modalidad de: un autor, dos autores, tres autores, cuatro autores y más de cuatro autores. Su análisis nos permitió averiguar si los investigadores constituyen unidades aisladas o equipos de trabajo, a la vez que la consolidación de los grupos. En la siguiente tabla observamos que la mayoría de los trabajos $(87,2 \%)$ están firmados por un solo autor, el 6,3\% de trabajos están firmados por dos autores, el 2,4\% están firmados por tres autores, el 1,6\% están firmados por cuatro autores, y el 2,5\% están firmados por más de cuatro autores (tabla XI).

\begin{tabular}{|c|c|c|c|}
\hline $\mathrm{N}^{\circ}$ Autores firmantes & & $\mathrm{N}^{0}$ Trabajos & $\%$ \\
\hline Firmados por un solo autor & & 1391 & 87,2 \\
\hline Firmados por dos autores & & 100 & 6,3 \\
\hline Firmados por tres autores & & 38 & 2,4 \\
\hline Firmados por cuatro autores & & 25 & 1,6 \\
\hline Firmados por más de cuatro autores & & 41 & 2,5 \\
\hline & TOTAL & 1595 & 100 \\
\hline
\end{tabular}

Tabla XI. Número de autores por trabajo publicado. 


\section{Institución}

Una vez identificada la producción a nivel "macro" (producción a nivel nacional) y a nivel "micro" (autores y grupos de autores), en este apartado presentamos la distribución de la investigación por instituciones, identificando el tipo de institución. Como era de esperar, atendiendo al tipo de institución, la mayoría de la investigación procede de las universidades, concretamente el 79,7\% (1.272 trabajos). Otros centros educativos protagonizan el 7,5\% (121 trabajos), las unidades de calidad y evaluación el 2\% (32 trabajos) y, el resto, el 10'7\%, procede de otras instituciones (ver tabla XII). Estos datos nos muestran una deficiencia en investigación realizada por los profesionales prácticos de la educación en todo el territorio nacional.

\begin{tabular}{|c|c|c|}
\hline Institución & $\mathbf{f}$ & $\%$ \\
\hline Universidad & 1.272 & 79,8 \\
\hline Otras & 170 & 10,7 \\
\hline Otro centro educativo & 121 & 7,5 \\
\hline Unidades de calidad y evaluación & 32 & 2 \\
\hline Centro de Investigación & 0 & 0 \\
\hline TOTAL & 1.595 & $100 \%$ \\
\hline
\end{tabular}

Tabla XII. Número de trabajos por Institución.

Dado que la mayor producción está en las universidades, presentamos en la siguiente tabla (tabla XIII, siguiente punto), donde se muestra la producción por Comunidad Autónoma, la producción universitaria por Comunidad; es decir, hemos agrupado los documentos de procedencia universitaria por comunidad. De este modo tenemos que las universidades más productivas han sido las de las comunidades de Madrid, Andalucía, Cataluña, Valencia y Galicia, dato que a excepción de Galicia correlaciona perfectamente con el número de universidades y profesorado universitario de cada comunidad.

Si comparamos estos datos con los obtenidos por la categoría autor, nos damos cuenta de que los autores más productivos no pertenecen a las comunidades más productivas, excepto en el caso de Galicia y Valencia. El índice sube sobremanera en Galicia porque en la universidad de la Coruña se encuentra un grupo de investigadores (5 personas) con una alta producción cada uno de ellos. La comunidad valenciana destaca por tener el autor más productivo de toda la muestra (22 trabajos) en la Universidad de Alicante.

\section{Comunidad Autónoma}

Como ya indicamos en el epígrafe anterior, hay una gran equivalencia entre los resultados institucionales y zona geográfica. Las comunidades autónomas más productivas a nivel general han sido Madrid, con el 19\% de la producción total (300 trabajos), Andalucía, con el 13,5\% (213 trabajos), Cataluña, con un 8\% (126 trabajos), 
Valencia, con 7,7\% (122), y Galicia con 5,9\% (90 trabajos). Como podemos observar en la tabla XIII, la producción procedente de otros centros (no universitarios) también es mayor en las comunidades de alta producción universitaria.

\begin{tabular}{lcccc}
\hline Comunidad Autónoma & Universidad & Otros Centros & TOTAL & \% \\
\hline Fuera de España & 207 & 128 & 335 & 21 \\
Madrid & 248 & 52 & 300 & 19 \\
Andalucía & 184 & 29 & 213 & 13,5 \\
Cataluña & 105 & 21 & 126 & 8 \\
Valencia & 101 & 22 & 123 & 7,7 \\
Galicia & 90 & 4 & 94 & 5,9 \\
Castilla y León & 61 & 14 & 75 & 4,8 \\
Asturias & 57 & 5 & 62 & 3,8 \\
Murcia & 40 & 6 & 46 & 2,8 \\
País Vasco & 33 & 10 & 43 & 2,6 \\
Aragón & 30 & 9 & 39 & 2,4 \\
Islas Canarias & 31 & 8 & 39 & 2,4 \\
Navarra & 24 & 1 & 25 & 1,5 \\
Islas Baleares & 23 & 1 & 24 & 1,4 \\
Castilla la Mancha & 18 & 8 & 26 & 1,6 \\
Extremadura & 13 & 2 & 15 & 0,9 \\
La Rioja & 4 & 2 & 6 & 0,4 \\
Cantabria & 3 & 1 & 4 & 0,3 \\
& 1.272 & 323 & $\mathbf{1 . 5 9 5}$ & $\mathbf{1 0 0}$ \\
\hline
\end{tabular}

Tabla XIII. Producción por Comunidad Autónoma y por universidad.

\section{Tema de investigación}

Uno de los objetivos principales de este estudio fue conocer sobre qué se ha investigando sobre rendimiento académico. Para ello se analizaron los objetivos de cada estudio. A partir de los resultados hicimos una clasificación temática en torno a la cual agrupamos cada documento. Estas categorías las organizamos en cuatro grandes dimensiones: 1) Estudios relacionados sobre definiciones conceptuales sobre fracaso y éxito escolar; diagnóstico e intervención para la mejora y éxito escolar; 2) Factores relacionados o determinantes del éxito y fracaso académico; 3) Incidencia de fracaso académico y políticas educativas; 4) Estudios de otras variables relacionadas con el rendimiento académico.

\begin{tabular}{|c|c|c|c|c|c|}
\hline \multicolumn{3}{|c|}{ TEMAS MÁS TRATADOS } & \multicolumn{3}{|c|}{ TEMAS MENOS TRATADOS } \\
\hline Objeto investigación & f & $\%$ & Temas & $\mathbf{f}$ & $\%$ \\
\hline \multicolumn{3}{|c|}{$\begin{array}{c}\text { CONCEPTO, DIAGNÓSTICO E } \\
\text { INTERVENCION } \\
\end{array}$} & \multicolumn{3}{|c|}{ POLITICAS E INCIDENCIA } \\
\hline Diagnóstico causal & 320 & 20 & Evaluación resultados & 76 & 4,7 \\
\hline Procedimientos Intervención & 188 & 11,7 & Políticas educativas & 26 & 1,6 \\
\hline Conceptualización & 115 & 7,2 & Identificación & 36 & 2,2 \\
\hline
\end{tabular}




\begin{tabular}{|c|c|c|c|c|c|}
\hline TOTAL & 623 & $39,1 \%$ & TOTAL & 138 & $8,6 \%$ \\
\hline \multicolumn{3}{|c|}{ FACTORES CAUSALES RELACIONADOS } & \multicolumn{3}{|c|}{ OTRAS VARIABLES RELACIONADAS } \\
\hline Contexto Escolar & 234 & 14,7 & Psicología Clínica & 116 & 7,2 \\
\hline Contexto Familiar & 106 & 6,6 & Metodológico & 24 & 1,5 \\
\hline Contexto Social & 94 & 5,8 & Género & 17 & 1 \\
\hline TOTAL & 434 & $27,2 \%$ & Lingüístico & 17 & 1 \\
\hline \multicolumn{3}{|c|}{ FACTORES DEL ALUMNADO } & Extraescolar & 13 & 0,8 \\
\hline Motivación & 85 & 5,3 & Tecnología educativa & 10 & 0,6 \\
\hline Estrategias Aprendizaje & 57 & 3,5 & Educación especial & 9 & 0,5 \\
\hline Salud física & 33 & 2 & $\begin{array}{r}\text { TOTAL } \\
\end{array}$ & 206 & $13 \%$ \\
\hline Actitudinal & 6 & 0,3 & & & \\
\hline Afectivo & 13 & 0,8 & & & \\
\hline TOTAL & 194 & $12,1 \%$ & & & \\
\hline
\end{tabular}

Tabla XIV. Productividad sobre rendimiento académico según el Contenido.

Los objetivos de la investigación del rendimiento académico en España han estado mayormente dirigidos a averiguar las causas del bajo/alto rendimiento $(39,3 \%)$, en todas sus dimensiones: sociales, familiares, escolares y personales. De este modo, no sólo se ha construido conocimiento sobre las causas del éxito y fracaso académico, sino que se ha ido dibujando la constelación de factores relacionados con el aprendizaje. Sin embargo, las publicaciones han estado más dirigidas a desarrollar y tratar modelos conceptuales, diagnósticos y de intervención (39,1\%). Otro lugar preferente lo ocupa la investigación y el estudio de las políticas educativas y su incidencia en el éxito escolar y el desarrollo educativo en general (8,6\%). Finalmente, encontramos un conjunto de trabajos cuyo foco de atención no es el rendimiento sino otras variables que han estudiado el rendimiento como variable relacionada (13\%).

\section{Conclusiones}

La valoración global de los resultados obtenidos tras el análisis de la literatura española sobre rendimiento académico de las tres últimas décadas nos muestra: un constante crecimiento de la investigación y una amplia variedad de variables analizadas en relación al éxito y fracaso académico; un uso de lenguaje adecuado y normalizado en la descripción del contenido a través de los títulos; una producción emanada básicamente de las universidades de todas las Comunidades Autónomas; y una gran dispersión de los trabajos, tanto entre autores, cuya mayoría sólo ha publicado un trabajo, como entre las fuente de publicación, donde sucede casi lo mismo.

A pesar del aparente volumen de producción (1.595 trabajos), en relación a otros ámbitos de interés de la investigación en educación, el "rendimiento académico" se muestra como un tema pobremente tratado, pues son muchos los estudios que lo abordan sólo de un modo secundario, en relación a otras variables, o como estudio teórico de modelos y propuestas de intervención. Se nota una escasez de estudios en profundidad más esclarecedores sobre los factores relacionados, así como ausencia de 
estudios de estos factores relacionados en poblaciones y contextos específicos, así como propuestas concretas para la mejora de estos grupos de interés.

En relación a la producción, se ha mantenido constante con un ligero ascenso en el tiempo, situándose en la última década el mayor número de publicaciones. Entendemos que esto se produce como consecuencia del incremento de la inversión en investigación de esta década, y la revalorización del conocimiento científico tanto dentro como fuera del territorio nacional, sin dejar de mencionar la incentivación de la producción a los investigadores por parte de la comisión nacional de evaluación de la actividad investigadora, así como los criterios de evaluación y acreditación del profesorado universitario español, sobre los que se ha ejercido una gran presión para obtener una mayor producción.

Las mayores debilidades de la producción sobre rendimiento las encontramos en la cantidad y dispersión de autores y soportes de publicación. En relación a los autores, hay escasez de grupos estables de trabajo. Por un lado, la mayoría de los autores han publicado un solo trabajo, lo que indica una investigación casual y poco especializada, y, por otro, los autores más pioneros y relevantes publican de modo individual, de lo que se deduce una ausencia de redes dentro y fuera del contexto institucional al que pertenecen. Con ello, se confirma la ley de Lotka sobre productividad de los autores, que indica la proporción inversa entre número de autores y número de trabajos que publican.

En relación al soporte de publicación, se constata una amplia producción de libros y capítulos de libros. Esta realidad, forma parte de una tradición en las ciencias de la educación, muchas veces por razones y finalidades divulgativas, pues los libros son los soportes más utilizados por los profesionales prácticos (profesorado y técnicos educativos). Sin embargo, las editoriales y revistas donde se alojan las publicaciones son en su mayoría poco especializadas en conocimiento educativo, y poco constantes en la publicación de la temática abordada (la media es de tres artículos por revista). La mayoría de las revistas y editoras publican un solo artículo o libro, lo que dificulta enormemente a los especialistas localizar el conocimiento. Esta realidad muestra una relación inversa entre la relevancia temática (pues el rendimiento ha constituido y constituye un ámbito de investigación de preocupación constante, tanto para los investigadores y profesionales como para familias y otros agentes sociales), y la ausencia de revistas especializadas, tanto en temáticas como en audiencias. Sin embargo, hay que destacar la presencia de un grupo de revistas de educación, de tipo multidisciplinar, que publican un gran número de trabajos sobre rendimiento, y están actualmente reconocidas por su prestigio y calidad, que cumplen los requisitos de normalización de las publicaciones científicas, se encuentran en el primer cuartil de los índices de impacto de los últimos años, y muchas de ellas forman parte del Journal Citation Report (JCR). Nos referimos, entre otras a: Revista de Investigación Educativa (RIE); Revista Bordón; Revista Española de Pedagogía; Revista de Educación; Revista Psicothema.

En otro orden, las publicaciones derivadas de tesis doctorales se muestran escasas, por lo que habría que hacer un análisis más exhaustivo que nos permita determinar el número de publicaciones por tesis doctoral, y romper con una mala práctica en 
educación que es la de ralentizar la publicación del conocimiento derivado de tesis doctorales.

Con el indicador "autor" comprobamos que en la producción de 1.595 documentos científicos han participado 1.941 autores, de los cuales el 82\% sólo ha publicado un trabajo (en algunos casos en colaboración). Sin embargo, sí se observa un grupo de investigadores constantes y altamente productivos. Llama la atención la correlación entre el grupo de autores que sólo han publicado un trabajo y única autoría; también el alto porcentaje de artículos con única autoría (87\%). Este dato pone de manifiesto un gran volumen de publicaciones protagonizadas por expertos que de un modo muy puntual han estudiado el rendimiento académico, y un grupo estable, constante, aunque menos integrados en equipos de trabajo. A nivel institucional y regional, no hay una relación entre las comunidades autónomas y universidades más productivas con los grupos o autores más productivos. En este sentido, vemos como las comunidades más productivas, junto con sus universidades, son las Comunidad de Madrid y la de Andalucía, seguidas de Cataluña y Valencia, mientras que los autores más productivos están en Alicante, La Coruña, Islas Baleares y Asturias. Estos datos desvelan un índice de productividad aceptable, y un escaso índice de colaboración y visibilidad del conocimiento en esta área. Se requiere de estudios de cienciometría posteriores, a partir de este, que permitan identificar con mayor precisión tendencias y lagunas en la producción y en las redes de construcción y difusión, a la vez que un análisis de los canales de difusión empleados, y si estos están siendo vehículos de transmisión hacia la socialización del conocimiento.

Este estudio ha supuesto sólo una aproximación a la literatura científica sobre el rendimiento académico en España. Con este primer paso hemos localizado, ordenado y caracterizado la producción, para posteriores investigaciones de síntesis de resultados y de evaluación de los procesos y la calidad de la investigación realizada sobre este ámbito. Hasta hace pocos años las ciencias sociales en general recibían poca atención de cómo se localizaban, se indexaban en sumarios, se evaluaban y se interpretaban los resultados de investigación. Actualmente, debido a alta producción y los requerimientos sociales a la producción científica, la fiabilidad de los hallazgos son una condición para la construcción del conocimiento, por lo que se vuelve imprescindible la evaluación de la investigación primaria, y la integración de los resultados por medio de estudios de síntesis. Por otro lado, las "prácticas basadas en la evidencia” han requerido conocer cómo se ha hecho la ciencia, qué hallazgos han tenido lugar, y qué mejores prácticas se sugieren. Desde estas perspectivas, este estudio supone no sólo una aproximación sintetizada a la producción sobre rendimiento académico, sino un paso previo a otros estudios de contenido que nos permitan integran conclusiones de los distintos autores, construir puentes entre los distintos hallazgos, e identificar problemas de investigación poco resueltos. 


\section{Referencias bibliográficas}

CAMPS, D. (2008). Limitaciones de los indicadores bibliométricos en la evaluación de la actividad científica biomédica. Redalyc, 39 (1), 74-79.

COOPER, H. (2007). Evaluation and interpreting research synthesis in adult learning and literacy. Boston: National College Transition Network, New England Literacy, Resorce Center/World Education.

COOPER, H. (2009). Research synthesis and meta-analysis: a step-by-step approach. Los Angeles, Sage.

FERNÁNDEZ Cano, A. (1995). Métodos para evaluar la investigación en psicopedagogía. Madrid: Síntesis.

GARVEY, W.D. AND GRIFFITH, B.C. (1971). Scientific communication: Its role in the conduct of research and creation of knowledge. American Psychologist, 26, 349-361.

KOENING, M. E. D. (1983). Bibliometric indicators versus expert opinion in assessing research performance. Journal of the American Society for Information Science, 34 (2), 136-145.

LÓPEZ PIÑEIRO, J.M. y TERRADA, M. L. (1993). Veinte años de investigación bibliométrica en el Instituto de Estudios Documentales e Históricos sobre la Ciencia. Cuadernos de Documentación e Informática Biomédica. Valencia.

LÓPEZ-LÓPEZ P. (1996). Introducción a la Bibliometría. Valencia: Promolibro.

LÓPEZ-LÓPEZ P. y TORTOSA Gil, F (2002). Los métodos Bibliométricos en Psicología. En Tortosa, F.M y Civera, C. (coords). Nuevas Tecnologías de la Información y Documentación en Psicología, 199-226. Editorial Ariel, Barcelona.

MALTRÁS BARBA, B., QUINTANILLA MIGUEL, A., y VIDAL, J. (1998). Indicadores bibliométricos en la evaluación de la investigación. Revista de Educación, 315, 141-151.

NEDERHOF, A. J. y VAN RAAN, A. F. J. (1987). Peer review and bibliometric indicators of scientific performance: a comparison of cum laude doctorates with ordinary doctorates in physics. Scientometrics, 11 (5-6), 333-350.

PEÑA OCANDO, D.; PORTILlO, L. y CALDERA, E. (2011). Indicadores de productividad, colaboración y circulación de la investigación. Caso: Escuela Bibliotecología y Archivología de Luz. Revista de Documentación de las Ciencias de la Información, 34, 291-306.

PERSSON, O., GLÄNZEL, W. \& DANELL, R (2004). Inflationary bibliometric values: The role of scientific collaboration and the need for relative indicators in evaluative studies. Scientometrics, 60 (3), 421-432.

QUEVEDO-BLASCO, R. y LÓPEZ-LÓPEZ, W. (2010). Análisis Bibliométrico de las Revistas Multidisciplinares de Psicología Recientemente Incorporadas en la Web of Science (2008-2009). Psicología: Reflexão e Crítica, 23, 384-408. 
SÁNCHEZ MECA, J. y BOTELLA, J. (2010). Revisiones Sistémicas y Meta-análisis: Herramientas para la práctica Profesional. Papeles del Psicólogo, 31, 7-17.

TAVEGGIA, T.C. (1974). Resolving research controversy through empirical cumulation. Sociological Methods and Research, 2, 395-407.

WARNER, J. (2000). A critical review of the application of citation studies to the Research Assessment Exercises. Journal of Information Science, 26 (6), 453-460.

\section{Correspondencia con la autora}

Lidia CABRERA PÉREZ

Facultad de Educación.

Avda Trinidad, s/n.

Campus Central. Universidad de La Laguna.

38204 La Laguna. Santa Cruz de Tenerife.

e-mail: dcabrera@ull.edu.es 